\title{
Development and Validation of Nomograms for Predicting Cancer-Specific Survival in Elderly Patients with Intrahepatic Cholangiocarcinoma After Liver Resection: A Competing Risk Analysis
}

This article was published in the following Dove Press journal:

Cancer Management and Research

\author{
Tao Wang ${ }^{1, *}$ \\ Jinfu Zhang ${ }^{1}{ }^{1, *}$ \\ Wanxiang Wang ${ }^{2}$ \\ Xianwei Yang' \\ Junjie Kong ${ }^{3}$ \\ Shu Shen ${ }^{\prime}{ }^{\prime}$ \\ Wentao Wang (D) \\ 'Department of Liver Surgery and Liver \\ Transplantation Center, West China \\ Hospital of Sichuan University, Chengdu, \\ People's Republic of China; ${ }^{2}$ Department \\ of Hepatobiliary, Pancreatic, and Splenic \\ Surgery, The Affiliated Hospital of Inner \\ Mongolia Medical University, Hohhot, \\ People's Republic of China; ${ }^{3}$ Department \\ of Liver Transplantation and \\ Hepatobiliary Surgery, Shandong \\ Provincial Hospital, Cheeloo College of \\ Medicine, Shandong University, Jinan, \\ Shandong Province, People's Republic of \\ China
}

*These authors contributed equally to this work
Correspondence: Wentao Wang Department of Liver Surgery and Liver Transplantation Center, West China Hospital of Sichuan University, Chengdu, People's Republic of China

Tel +86 I898060I895

Fax +86-28-8542287।

Email wwtdoctor02@163.com
Background: There are few studies on the prognosis of elderly intrahepatic cholangiocarcinoma (iCCA) patients after liver resection. The aims of this study were to assess the cumulative incidences of cancer-specific mortality in elderly iCCA patients and to construct a corresponding competing risk nomogram for elderly iCCA patients.

Methods: We performed a retrospective analysis of elderly patients with iCCA who underwent liver resection between January 2006 and December 2019. Eligible elderly iCCA patients were randomly divided into training and validation sets at a ratio of $7: 3$. Based on the results of multivariate analysis using the Fine-Gray competing risk model, we developed a competing risk nomogram using data from the training set to predict the cumulative probabilities of iCCA-specific mortality. The performance of the nomogram was measured by the concordance index (C-index) and calibration curves. To evaluate the clinical usefulness of the nomogram, the clinical benefit was measured by using decision curve analysis (DCA). Furthermore, the patients were categorized into two groups according to the dichotomy values of the nomogram-based scores, and their survival differences were assessed using Kaplan-Meier and cumulative incidence function (CIF) curves.

Results: The 1-year, 3-year and 5-year cumulative iCCA-specific mortalities were $19.7 \%$, $48.3 \%$ and $56.1 \%$, respectively, for elderly iCCA patients. The multivariate Fine-Gray analysis indicated that microvascular invasion, macroscopic vascular invasion and lymph node metastasis were related to a significantly higher likelihood of iCCA specific mortality. The established nomogram was well calibrated and had a good discriminative ability, with a concordance index (C-index) of 0.742 (95\% CI, 0.708-0.748). Furthermore, the DCA indicated that the nomogram had positive net benefits compared with the conventional staging systems. In the training set and validation sets, the high-risk group had the higher probabilities of iCCA cancer-specific mortality than the low-risk group; meanwhile, the patients in the high-risk the group had significantly poorer overall survival (OS) than those in the low-risk group.

Conclusion: Elderly iCCA patients had comparable long-term outcomes with non-elderly iCCA patients. In addition, we constructed a prognostic nomogram for predicting survival in elderly iCCA patients based on the competing risk analysis. The competing risk nomogram displayed excellent discrimination and calibration.

Keywords: intrahepatic cholangiocarcinoma, iCCA, liver resection, elderly patients, competing risk analysis, nomogram 


\section{Background}

Intrahepatic cholangiocarcinoma (iCCA) is the second most common primary liver cancer after hepatocellular carcinoma (HCC). ${ }^{1}$ As the incidence and related mortality of iCCA are dramatically increasing worldwide, iCCA has become the focus of increasing concern., ${ }^{2,3}$ Despite improvements in iCCA surveillance and in the application of targeted therapy and immunotherapy, iCCA patients, especially in elderly patients, still have poor survival rates. ${ }^{4}$ Surgical resection remains the mainstay of potentially curative therapy for iCCA. ${ }^{5-7}$ As the aging of the population intensifies, the number of elderly iCCA patients has also increased dramatically. It was worth noting that the incidence of iCCA increases with age, and elderly patients tend to have a higher incidence of iCCA than younger patients. ${ }^{8}$ Elderly patients often have a higher incidence of chronic disease, worse health status, and organ dysfunctions. It is also undetermined whether these elderly patients benefit substantially more from surgical therapies. Therefore, it is necessary to comprehensively evaluate the safety of surgery and prognosis of elderly patients with iCCA.

Although there have been some reports of risk factors associated with elderly iCCA patient survival, most of them were based on Cox proportional hazards regression models and Kaplan-Meier estimates. ${ }^{9,10}$ The above method does not take into account competing risk events when is performed; thus, the probabilities of iCCAspecific death would be overestimated. ${ }^{11,12}$ In this context, the competing risk model is superior to the conventional methods because it takes into consideration competing events and can differentiate between the effects of therapy and risk factors on specific events without the assumption of independence between event types. ${ }^{13}$ Considering the high risk of competing events (various comorbidities) in elderly patients, ${ }^{4,14}$ it is essential to take other causespecific mortality into account when performing survival analysis for elderly iCCA patients. To date, no studies have adopted a competing risk model to examine the factors influencing the prognosis of elderly patients with iCCA.

Therefore, a competing risk analysis was performed to determine the predictive factors for iCCA-specific mortality in elderly patients. We developed a nomogram to offer clinicians a quantitative means to assess the individual cumulative incidences of iCCA-specific mortality to improve clinical decision making.

\section{Patients and Methods}

\section{Patients and Study Design}

In accordance with the definition of the World Health Organization, elderly patients were defined as those aged 60 years or older. ${ }^{15} \mathrm{~A}$ total of 328 elderly patients $(\geq 60$ years) who underwent liver resection for iCCA between January 2006 and December 2019 at West China Hospital were enrolled in this study. Our selection criteria for patients in this study included the following (1) patients aged $\geq 60$ years; (2) patients who underwent curative liver resection, with tumor tissues pathologically confirmed as iCCA, which was defined as the complete removal of all macroscopic nodules with a clear margin (R0 resection); (3) patients with Child-Pugh A or B7 (score $\leq 7$ ) liver function; and (4) patients with detailed clinical characteristics. Our exclusion criteria for this study were as follows: (1) patients with postoperative pathology confirmed R1 excision or tumor margin that was not specified in detail; (2) patients with a history of other extrahepatic malignancies. (3) Poor clinical data integrity. We used data from non-elderly people (age $<60$ years) in the same period to explore the difference in prognosis between non-elderly and elderly iCCA patients after liver resection. To overcome possible selection bias between the elderly group and non-elderly group, we used the method of propensity score matching (PSM) analysis according to sex, hepatitis B surface antigen ( $\mathrm{HBsAg}$ ), portal hypertension, tumor size, tumor number, microvascular invasion (MVI), macroscopic vascular invasion (MCI), satellite nodules, lymph node metastasis (LNM), tumor location and extent of liver resection to randomly match patients with nonelderly groups (age $<60$ years) as a control group to patients with elderly patients at a 1:1 ratio by using the "nearest neighbor" method with a caliper of $0.02 .{ }^{16}$ In addition, the whole group (age $\geq 60$ years) was randomly divided into two groups, $230(70 \%)$ were in the training set and 98 $(30 \%)$ were in the validation set. The training set was used to develop the nomograms for predicting the prognosis of elderly iCCA patients, whereas the validation set was used to verify the models. The flowchart of this present study process is shown in Figure 1 and the clinicopathologic characteristics of the patients in the training and validation sets are listed in Table 1. The study was approved by ethics committee of Sichuan University West China hospital and informed consent was taken from all the patients. The study was conducted in accordance with the Declaration of Helsinki. 


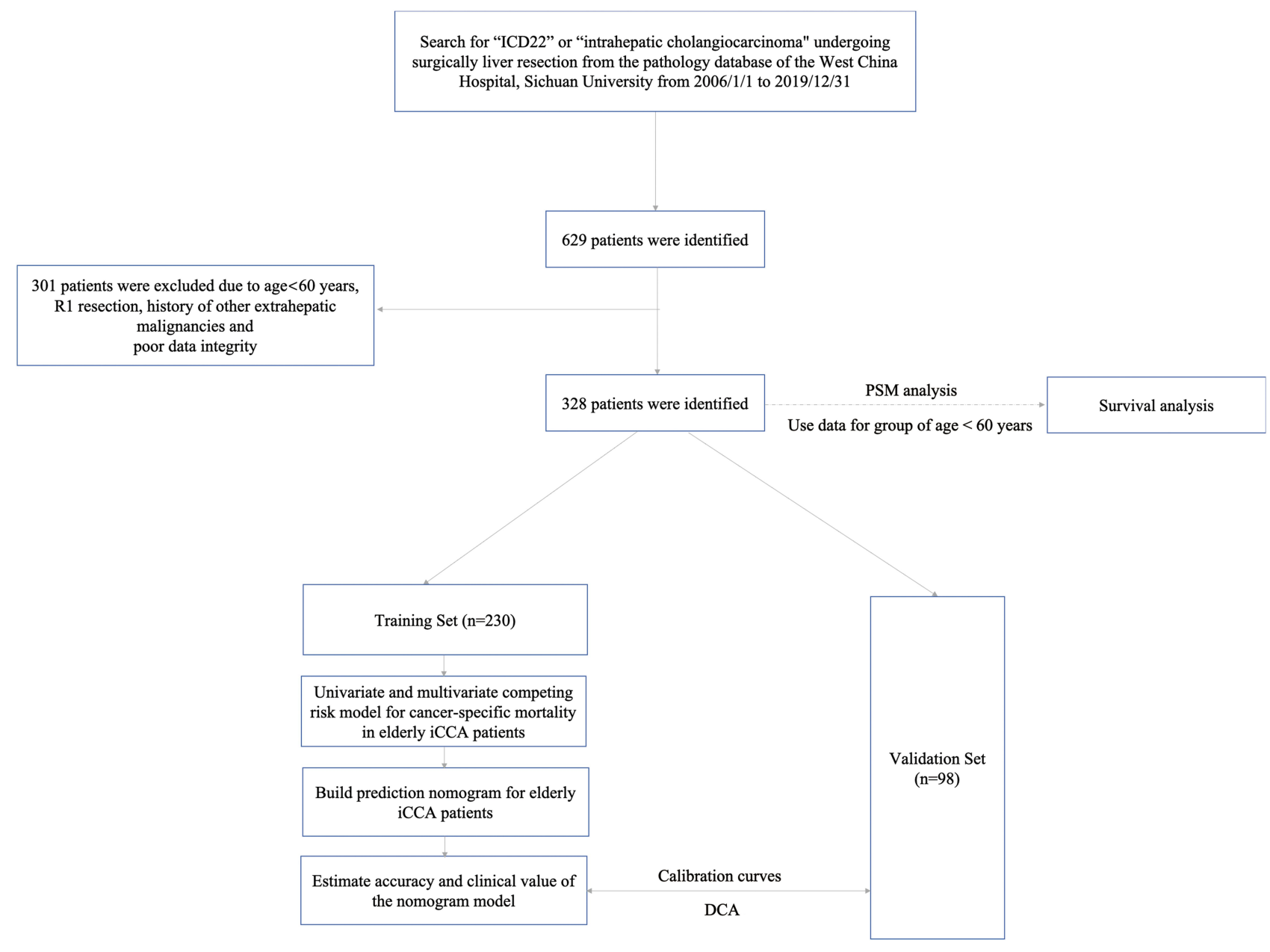

Figure I Flowchart of the patient selection process.

\section{Data Collection and Definitions}

Clinical data were gathered for all patients with iCCA including demographics, preoperative serum biochemistry data, preoperative serum tumor markers, imaging characteristics of tumors, histological reports, 8th American Joint Committee on Cancer (AJCC) TNM stage, ${ }^{17}$ Okabayashi stage, ${ }^{18}$ the Liver Cancer Study Group of Japan (LCSGJ) stage. ${ }^{19}$ Portal hypertension was defined as the presence of either esophageal varices or splenomegaly with a decreased platelet count $\left(100 \times 10^{9} / \mathrm{L}\right.$ or less). Macroscopic vascular invasion included major hepatic vessel invasion, defined as invasion of the firstand second-order branches of the portal veins or hepatic arteries, or as invasion of one or more of the three hepatic veins. Major resection was defined as resection of 3 or more Couinaud segments, while minor resection was defined as resection of fewer than 3 Couinaud segments. ${ }^{20}$ Postoperative surgical complications were defined according to the Clavien-Dindo classification. ${ }^{21}$ Prognostic nutritional index (PNI) was used to assess the nutritional status of patients. It is based on the serum albumin concentration and absolute lymphocyte count; $\mathrm{PNI}=$ serum albumin concentration $(\mathrm{g} / \mathrm{L})+5 \times$ total lymphocyte count $\left(\times 10^{9} / \mathrm{L}\right) .{ }^{22}$ The patients were classified into "PNI-low" (PNI $<45)$ and "PNI-high" (PNI $\geq 45$ ) groups as reported previously. ${ }^{23,24}$ We used the Eastern Cooperative Oncology Group (ECOG) score to assess the physical status of each iCCA patients, In general, the ECOG score of iCCA patients who underwent liver resection could not exceed 2 points. ${ }^{25}$

\section{Follow-Up and Recurrence Treatment}

In general, all patients who received liver resection were prospectively followed up through outpatient clinic visits or phone calls at intervals of 2-3 months during the first year after the operation and 3-6 months later. The diagnosis of recurrence was based on CT or MRI imaging findings, increased serum carbohydrate antigen19-9 (CA19-9) levels. Chest CT examination and bone scintigraphy were performed when extrahepatic tumor 
Table I The Comparison of Clinicopathological Factors Between Training Set and Validation Set

\begin{tabular}{|c|c|c|c|}
\hline \multirow[t]{2}{*}{ Characteristics } & \multicolumn{2}{|l|}{ Patients } & \multirow[t]{2}{*}{$P$ value } \\
\hline & Training Set $(n=230)$ & Validation Set(n=98) & \\
\hline Age (years), median (IQR) & $63(61-67.25)$ & $63(61-69.25)$ & 0.924 \\
\hline Gender, (male/female) & $136 / 94$ & $54 / 44$ & 0.499 \\
\hline HbsAg positive, n (\%) & $82(35.7 \%)$ & $29(29.6 \%)$ & 0.288 \\
\hline Portal hypertension, n (\%) & $45(19.6 \%)$ & $15(15.3 \% \%)$ & 0.361 \\
\hline \multicolumn{4}{|l|}{ Baseline laboratory investigations } \\
\hline WBC count $\times 10^{9} / \mathrm{L}$, median (IQR) & $6.2(5.20-7.60)$ & $5.81(4.93-7.63)$ & 0.603 \\
\hline NEUT count $\times 10^{9} / \mathrm{L}$, median (IQR) & $4.04(3.11-5.36)$ & $3.845(2.88-5.34)$ & 0.381 \\
\hline PLT count $\times 10^{9} / \mathrm{L}$, median (IQR) & $148(\mid 12-193)$ & $157.5(|| 2-2 \mid 7.5)$ & 0.461 \\
\hline ALT (U/L), median (IQR) & $25.5(17-39)$ & $24(16-32)$ & 0.139 \\
\hline AST (U/L), median (IQR) & $30(23-40)$ & $29.5(24-36.5)$ & 0.671 \\
\hline GGT (U/L), median (IQR) & $69(36.75-125.75)$ & $29.5(24-36.25)$ & 0.962 \\
\hline TBIL $(\mu \mathrm{mol} / \mathrm{L})$, median $(\mathrm{IQR})$ & $10.9(6.8-14.8)$ & $9.95(5.65-16.3)$ & 0.381 \\
\hline ALB $(g / L)$, median (IQR) & $42.25(39.3-45.2)$ & $42.2(38.75-45.03)$ & 0.516 \\
\hline PT(s), median (IQR) & $11.7(11.1-12.3)$ & $11.5(11.1-12.1)$ & 0.335 \\
\hline INR, median (IQR) & $1.03(0.97-1.09)$ & $1.02(0.96-1.08)$ & 0.372 \\
\hline CAI9-9 level(U/mL), median (IQR) & $76.2(19.1-405.5)$ & $49.75(15.58-463.78)$ & 0.529 \\
\hline PNI & & & 0.518 \\
\hline$\geq 45$ & $190(82.6 \%)$ & $78(79.6 \%)$ & \\
\hline$<45$ & $40(17.4 \%)$ & $20(20.4 \%)$ & \\
\hline Tumor size $(\mathrm{cm})$, median (range) & $5.3(3.875-7.125)$ & $4.9(1.5-13)$ & 0.348 \\
\hline Tumor number, (Multiple/solitary) & & & 0.329 \\
\hline Multiple & $71(30.9 \%)$ & $25(25.5 \%)$ & \\
\hline Solitary & $159(69.1 \%)$ & $73(74.5 \%)$ & \\
\hline Tumor location, n(\%) & & & 0.714 \\
\hline Left lobe & $88(38.3 \%)$ & $39(39.8 \%)$ & \\
\hline Right lobe & $88(38.3 \%)$ & $40(40.8 \%)$ & \\
\hline Both lobes & $54(23.4 \%)$ & $19(19.4 \%)$ & \\
\hline Extent of liver resection, $n(\%)$ & & & 0.106 \\
\hline Major & $12 \mid(52.6 \%)$ & $42(42.9 \%)$ & \\
\hline Minor & $109(47.4 \%)$ & $56(57.1 \%)$ & \\
\hline MVI, n (\%) & & & 0.279 \\
\hline Yes & $42(18.26 \%)$ & $23(23.47 \%)$ & \\
\hline No & $188(81.74 \%)$ & $75(76.53 \%)$ & \\
\hline Macroscopic vascular invasion, $n(\%)$ & & & 0.996 \\
\hline Yes & $68(29.57 \%)$ & $29(29.59 \%)$ & \\
\hline No & $162(70.43 \%)$ & $69(70.41 \%)$ & \\
\hline Satellite nodules, $n(\%)$ & & & 0.959 \\
\hline Yes & $31(13.5 \%)$ & $13(13.3 \%)$ & \\
\hline No & 199(86.5\%) & $85(86.7 \%)$ & \\
\hline Lymph node metastasis, n (\%) & & & 0.384 \\
\hline Present & $53(23.0 \%)$ & $27(27.55 \%)$ & \\
\hline Absent & 177 (77.0\%) & $71(72.45 \%)$ & \\
\hline Tumor encapsulation, $n(\%)$ & & & 0.697 \\
\hline Incomplete & $119(51.7 \%)$ & $53(54.1 \%)$ & \\
\hline Complete & III (48.3\%) & $45(45.9 \%)$ & \\
\hline
\end{tabular}

(Continued) 
Table I (Continued).

\begin{tabular}{|c|c|c|c|}
\hline \multirow[t]{2}{*}{ Characteristics } & \multicolumn{2}{|l|}{ Patients } & \multirow[t]{2}{*}{$P$ value } \\
\hline & Training Set $(n=230)$ & Validation Set(n=98) & \\
\hline Operation approach, n(\%) & & & 0.394 \\
\hline LLR & $19(8.3 \%)$ & $\mathrm{II}(\mathrm{II.2 \% )}$ & \\
\hline OLR & $211(91.7 \%)$ & $87(88.8 \%)$ & \\
\hline Complication, Clavien-Dindo $\geq 3, n$ (\%) & & & 0.600 \\
\hline Yes & $28(12.2 \%)$ & $14(14.3 \%)$ & \\
\hline No & $202(87.8 \%)$ & $84(85.7 \%)$ & \\
\hline Survival status & & & 0.157 \\
\hline Cancer-specific death & III(48.3\%) & $63(64.3 \%)$ & \\
\hline Non-cancer-specific death & $27(11.7 \%)$ & $8(8.2 \%)$ & \\
\hline Alive & $92(40 \%)$ & $27(27.5 \%)$ & \\
\hline
\end{tabular}

Abbreviations: $\mathrm{HBsAg}$, hepatitis B surface antigen; WBC, white blood cell; NEU, neutrophil; PLT, platelet; ALT, alanine aminotransferase; AST, aspartate transaminase; GGT, $\gamma$-glutamyl transferase; TBIL, total bilirubin; ALB, albumin; PT, prothrombin time; INR, international normalized ratio; CAI9-9, carbohydrate antigen I9-9; PNI, Prognostic Nutritional Index; MVI, microvascular invasion; LLR, laparoscopic liver resection; OLR, open liver resection.

recurrence was suspected. Survival information, including cancer-specific survival (CSS), OS, CSS was collected until May 31, 2020. OS was defined as the interval between resection and death, or the period up to the observation point. CSS referred to the duration from diagnosis to death from iCCA or tumor recurrence, patients who were alive at the point of last follow-up were considered as censored events.

\section{Statistical Analysis}

Continuous variable data are expressed as median and interquartile ranges (Q1-Q3). Categorical data are expressed as numbers and percentages. For comparisons between the different groups, the Chi-square test was used for categorical variables, and the Mann-Whitney $U$-test was used for continuous variables. First, the PSM analysis model was used to eliminate possible selection bias and increase the evidence level of this retrospective study. Second, considering that death from other causes might be a competitive event of elderly patients with iCCArelated death, we regarded other causes of death other than ICCA as competing events in our analysis of competing risks. Fine and Grey's models were adopted to evaluate the cumulative incidence function (CIF) of the variables on cancer-specific mortality and non-cancer-specific mortality. ${ }^{26,27}$ Univariate analysis was performed using the CIF to show the probability of each event and Gray's test to estimate the difference in the CIF between groups. ${ }^{12}$ Multivariate analysis with the Fine-Gray model was used to identify factors affecting the cumulative incidence of
iCCA. Hazard ratio (HR) and the associated 95\% confidence interval (CI) were calculated. We also compared the results from a Cox regression model with those from the Fine-Gray model.

A competing risk nomogram was built on the basis of the Fine and Grey's model. The discrimination and calibration power are two important aspects of the performance of the established nomograms and they were evaluated by the concordance index (C-index) and calibration curves, respectively. ${ }^{28,29}$ The $\mathrm{C}$-index reflects the probability of changes in the predicted survival along with the variation in predicted scores. The larger the $\mathrm{C}$-index is, the more accurate the nomogram is in predicting the prognosis. Moreover, in order to reduce the overfitting, calibration was evaluated by comparing the actual probabilities and the plot of the nomogram using 1,000 bootstrap samples. Finally, decision curve analysis (DCA) was conducted to assess the clinical usefulness and net benefit of the competing risk model. ${ }^{30,31}$ To determine whether the nomogram could successfully distinguish high-risk from low-risk elderly iCCA patients, each patient's prediction score was derived according to the nomogram, and the patients were categorized into the high-risk and low-risk groups based on the dichotomy values of the risk scores. Subsequently, the Kaplan-Meier and corresponding CIF curves of the two groups were plotted for the training set and validation set.

All statistical analyses were performed using, SPSS 25.0 for Mac (SPSS Inc, Chicago, IL, USA), Empower Stats (version 2018-12-22; http://www.empowerstats.com/cn/), 
and R statistical software (version 4.0.0; https://www.r-pro ject.org/). All statistical tests were two-sided, with $P<0.05$ considered to be indicative of statistical significance.

\section{Results}

\section{Patient Characteristics}

A total of 328 elderly patients with iCCA (age $\geq 60$ ) who underwent liver resection who met the inclusion criteria were enrolled in this study. The included patients had a median age of 63 years (Q1-Q3, 61-67 years), and $57.9 \%$ were male. The median follow-up time was 18.1 months (range 1-112 months). In total, 209/358 (58.4\%) patients died, $174(53.0 \%)$ cancer-specific deaths and 35 (10.7\%) non-cancer-specific deaths were observed. Among the 35 patients who died of noncancer-specific death, 8 patients died of sudden cardiovascular disease, 7 patients died of fractures caused by slipping or falls, 3 patients died of stroke, 4 patients died of severe respiratory diseases, and 13 patients died of unexplained accidental disease (no evidence of tumor recurrence or poor condition caused by cancer before death). The 1-year, 3-year, and 5-year iCCA cancer-specific mortality rates were $19.7 \%, 48.3 \%$ and $56.1 \%$, respectively. The baseline characteristics of the two different age groups differed before PSM, After PSM, 70 patients were included in each group, and the baseline characteristics of the patients in the two groups were comparable (listed in Supplementary Table 1). In addition, there were no obvious differences between the groups in OS or CSS before and after PSM analysis, as shown in Supplementary Figure S1. The demographic and clinical characteristics of the patients in the training and validation sets are listed in Table 1. The baseline demographic and clinical characteristics of the patients in the training and validation sets were similar $(P>0.05)$.

The univariate analysis included Gray's test and the CIF. When competing risks were present, the results of Gray's test showed that CA19-9, maximum tumor size, tumor number, MVI, macroscopic vascular invasion (MCI), satellite nodules, LNM exerted statistically significant effects on iCCA $(P<0.05)$. The CIF for almost all variables increased over 1,3 , and 5 years. The corresponding CIF curves were shown in Figure 2 and the data are listed in detail in Table 2. When competing events were present, we included statistically significant variables in the univariate analysis in the Fine-Gray model into the multivariate analysis. The multivariate analysis indicated that MVI (presence vs absence, HR, 1.843, 95\% CI, 1.138-2.962), MCI (presence vs absence, HR, 2.405, 95\% CI, 1.602-3.591) and LNM (Yes vs no, HR, $1.796,95 \%$ CI, 1.141-2.823) were significantly associated with a higher likelihood of iCCA specific mortality. Meanwhile, we compared the results of multivariate Cox regression with the results of Gray's test. Different from Fine-Gray multivariate analysis, multivariate Cox regression analysis identified that age $(\geq 70$ vs $<70$ years, HR, 1.706, 95\% CI, 1.101-2.645), MVI (presence vs absence, HR, 1.699, 95\% CI, 1.093-2.641), MCI (presence vs absence, HR, 2.398, 95\% CI, 1.648-3.489), and LNM (yes vs no, HR, 4.059, 95\% CI, 2.733-6.028) were independent predictors in patients after liver resection for iCCA, the data are listed in detail in Supplementary Table 2 and Table 3. Based on the results of the multivariate competing risk model analysis, a nomogram integrating all significant independent factors were constructed to calculate the 1-year, 3-year and 5-year cumulative iCCA cancerspecific mortality probabilities (Figure 3 ). The C-index of prediction of cancer specific survival in training set were 0.728 (95\% CI, 0.708-0.748) and 0.683 (95\% CI, 0.659-$0.721)$, respectively. The calibration plot was close to the 45-degree diagonal line, indicating that there was optimal agreement between the nomogram prediction and actual observations in the training set, as shown in Figure 4. Furthermore, we found that for our nomogram, the clinical net benefit gained from the competing risk model was higher than that from the Okabayashi staging system, the LCSGJ staging system and the 8th edition AJCC staging system in DCA, as shown in Figure 4. According to the dichotomy values of the nomogram-based scores derived from the training set, the patients were categorized into high-risk and low-risk groups in the training set and validation set. Kaplan-Meier analysis showed that the OS rates in high-risk group were significantly lower than those in the low-risk groups in the training set and validation set. (both $P<0.001)$ The high-risk group had the higher probabilities of iCCA cancer-specific mortality than the low-risk group in the training set and validation set (both $P<0.001$ ), as shown in Figure 5. Therefore, when using the nomogram as a predictive tool, clinicians could successfully discriminate among different risk groups.

\section{Discussion}

In medical research, the research objects usually do not experience only one type of event; instead, multiple endpoints that compete with each other are commonly present, that is, in the form of a competing event. Most medical research generally has competing risks. When discussing 
A

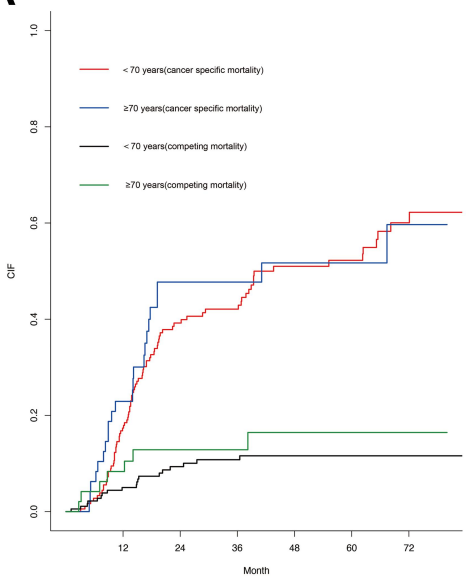

D

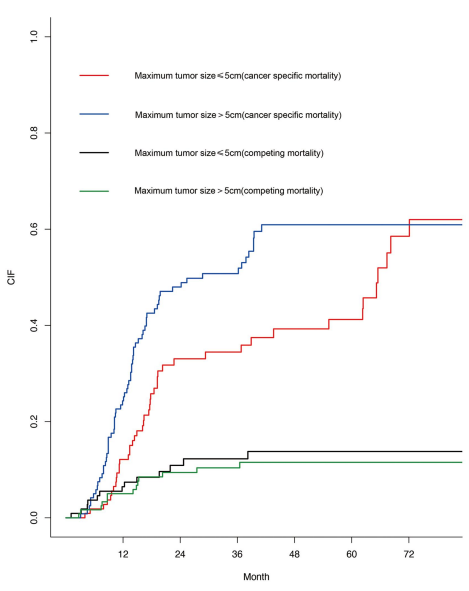

G

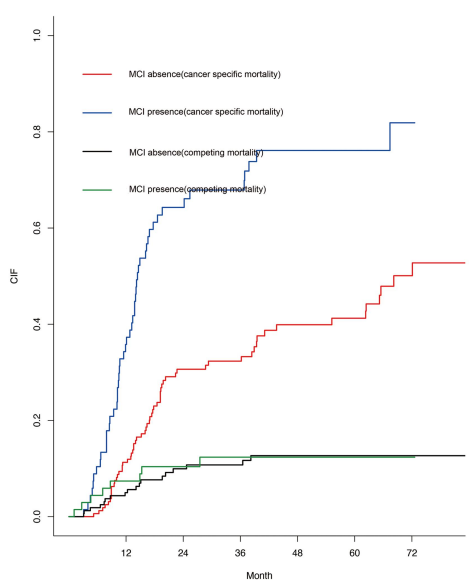

B

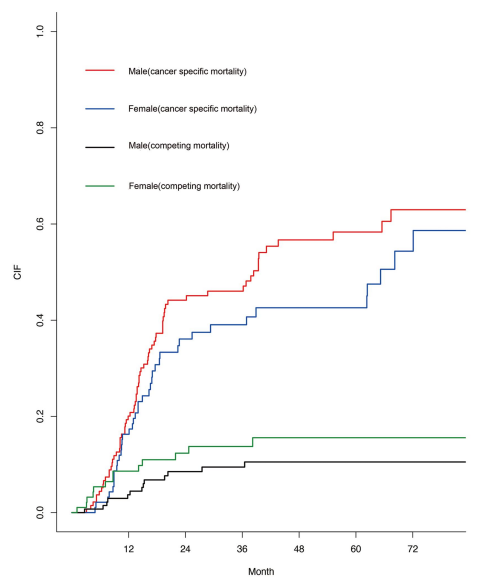

E

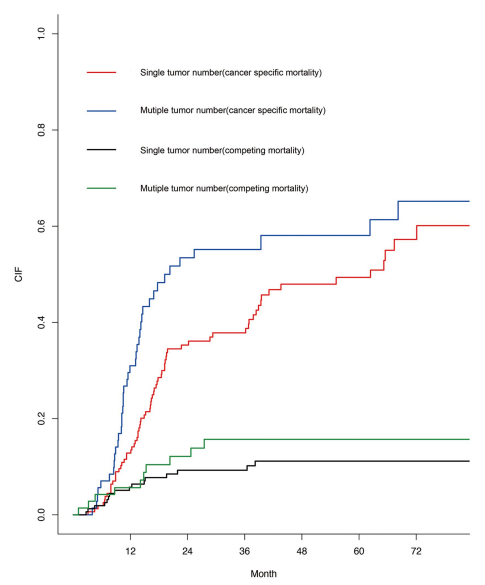

H

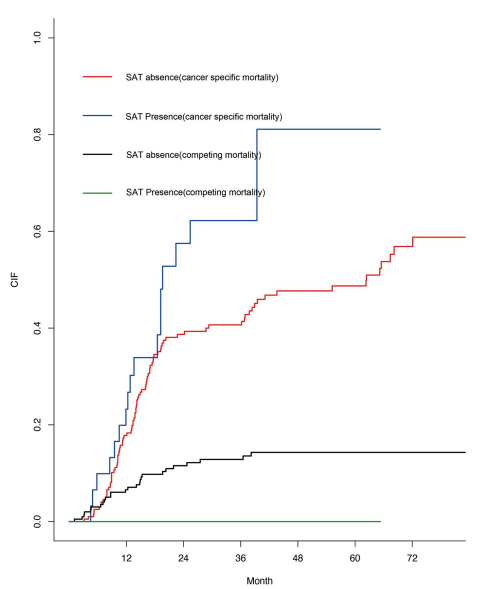

C

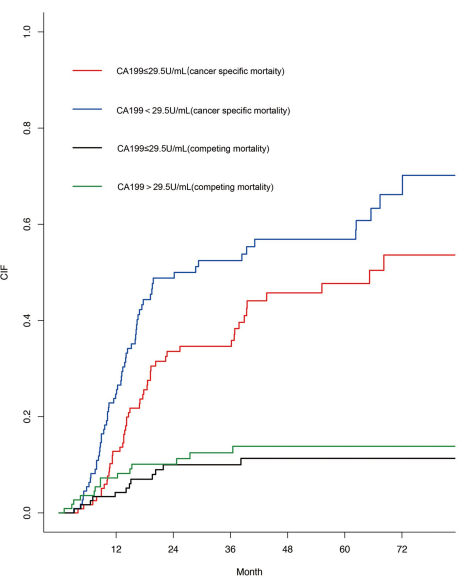

$\mathbf{F}$

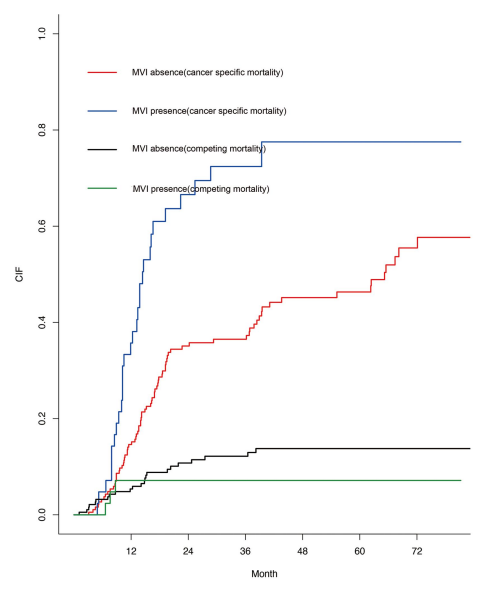

I

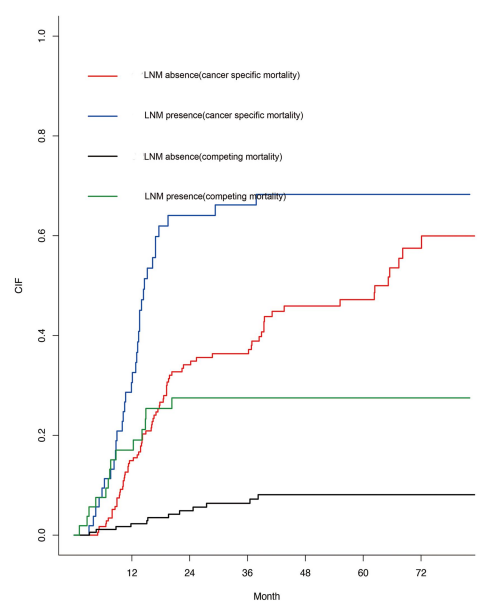

Figure 2 Cumulative cancer-specific and competing mortality curves for elderly iCCA patients stratified by the following patient characteristics: (A) age; (B) sex; (C) CA 99; (D) maximum tumor size; (E) tumor number; (F) MVI status; (G) MCl status; (H) SAT status; (I) LNM status.

Abbreviations: CA19-9, carbohydrate antigen 19-9; MVI, microvascular invasion; MCl, macroscopic vascular invasion; SAT, satellite nodules; LNM, lymph node metastasis.

specific causes of death, the occurrence of competing events affects the analysis of end events and may overestimate the cumulative incidence of each variable using the traditional analysis method (including Kaplan-Meier estimates of survival curves and Cox regression analyses). ${ }^{32-34}$ iCCA usually has locally aggressive behaviors, such as lymph node involvement, intrahepatic metastasis, peritoneal dissemination, and vascular 
Table 2 Univariable Analysis in Elderly Patients with iCCA by Using Competing Risk Model in the Training Set

\begin{tabular}{|c|c|c|c|c|c|}
\hline \multirow[t]{2}{*}{ Variables } & \multirow[t]{2}{*}{ Gray's Test } & \multirow[t]{2}{*}{$P$-value } & \multirow[b]{2}{*}{ I2-mo } & \multicolumn{2}{|c|}{ Cumulative Incidence Function } \\
\hline & & & & 36-mo & 60-mo \\
\hline $\begin{array}{l}\text { Age } \\
\qquad 770 \text { years } \\
<70 \text { years }\end{array}$ & 0.119 & 0.729 & $\begin{array}{l}0.229 \\
0.174\end{array}$ & $\begin{array}{l}0.477 \\
0.421\end{array}$ & $\begin{array}{l}0.517 \\
0.522\end{array}$ \\
\hline $\begin{array}{l}\text { Sex } \\
\text { Male } \\
\text { Female }\end{array}$ & 1.665 & 0.197 & $\begin{array}{l}0.201 \\
0.163\end{array}$ & $\begin{array}{l}0.460 \\
0.391\end{array}$ & $\begin{array}{l}0.583 \\
0.425\end{array}$ \\
\hline $\begin{array}{l}\text { Child-pugh score } \\
\text { B } \\
\text { A }\end{array}$ & 0.745 & 0.388 & $\begin{array}{l}0.250 \\
0.180\end{array}$ & $\begin{array}{l}0.250 \\
0.444\end{array}$ & $\begin{array}{l}0.594 \\
0.525\end{array}$ \\
\hline $\begin{array}{l}\text { HbsAg } \\
\text { Positive } \\
\text { Negative }\end{array}$ & 0.224 & 0.636 & $\begin{array}{l}0.212 \\
0.171\end{array}$ & $\begin{array}{l}0.418 \\
0.436\end{array}$ & $\begin{array}{l}0.529 \\
0.518\end{array}$ \\
\hline $\begin{array}{l}\text { Portal hypertension } \\
\text { Present } \\
\text { Absent }\end{array}$ & 2.649 & 0.104 & $\begin{array}{l}0.136 \\
0.230\end{array}$ & $\begin{array}{l}0.318 \\
0.460\end{array}$ & $\begin{array}{l}0.415 \\
0.546\end{array}$ \\
\hline $\begin{array}{l}\text { CA 19-9 level } \\
\begin{array}{l}>79 \mathrm{U} / \mathrm{mL} \\
\leq 79 \mathrm{U} / \mathrm{mL}\end{array}\end{array}$ & 6.672 & 0.010 & $\begin{array}{l}0.247 \\
0.128\end{array}$ & $\begin{array}{l}0.524 \\
0.346\end{array}$ & $\begin{array}{l}0.569 \\
0.477\end{array}$ \\
\hline $\begin{array}{l}\text { Maximum tumor size } \\
>5 \mathrm{~cm} \\
\leq 5 \mathrm{~cm}\end{array}$ & 5.653 & 0.017 & $\begin{array}{l}0.243 \\
0.121\end{array}$ & $\begin{array}{l}0.508 \\
0.344\end{array}$ & $\begin{array}{l}0.609 \\
0.412\end{array}$ \\
\hline $\begin{array}{l}\text { Tumor number } \\
\text { Multiple } \\
\text { Single }\end{array}$ & 4.721 & 0.030 & $\begin{array}{l}0.310 \\
0.128\end{array}$ & $\begin{array}{l}0.552 \\
0.378\end{array}$ & $\begin{array}{l}0.581 \\
0.493\end{array}$ \\
\hline $\begin{array}{l}\text { Tumor encapsulation } \\
\text { Incomplete } \\
\text { Complete }\end{array}$ & 1.575 & 0.209 & $\begin{array}{l}0.202 \\
0.167\end{array}$ & $\begin{array}{l}0.508 \\
0.348\end{array}$ & $\begin{array}{l}0.547 \\
0.489\end{array}$ \\
\hline $\begin{array}{l}\text { MVI } \\
\text { Yes } \\
\text { No }\end{array}$ & 19.429 & $<0.001$ & $\begin{array}{l}0.357 \\
0.146\end{array}$ & $\begin{array}{l}0.724 \\
0.365\end{array}$ & $\begin{array}{l}0.775 \\
0.463\end{array}$ \\
\hline $\begin{array}{l}\text { Macroscopic vascular invasion } \\
\text { Yes } \\
\text { No }\end{array}$ & 35.591 & $<0.001$ & $\begin{array}{l}0.358 \\
0.113\end{array}$ & $\begin{array}{l}0.679 \\
0.323\end{array}$ & $\begin{array}{l}0.761 \\
0.412\end{array}$ \\
\hline $\begin{array}{l}\text { Satellite nodules } \\
\text { Yes } \\
\text { No }\end{array}$ & 4.260 & 0.039 & $\begin{array}{l}0.232 \\
0.178\end{array}$ & $\begin{array}{l}0.622 \\
0.407\end{array}$ & $\begin{array}{l}0.811 \\
0.487\end{array}$ \\
\hline $\begin{array}{l}\text { Lymph node metastasis } \\
\text { Yes } \\
\text { No }\end{array}$ & 7.687 & $<0.001$ & $\begin{array}{l}0.306 \\
0.149\end{array}$ & $\begin{array}{l}0.662 \\
0.363\end{array}$ & $\begin{array}{l}0.683 \\
0.472\end{array}$ \\
\hline $\begin{array}{l}\text { Extent of liver resection } \\
\text { Major } \\
\text { Minor }\end{array}$ & 0.243 & 0.622 & $\begin{array}{l}0.226 \\
0.140\end{array}$ & $\begin{array}{l}0.442 \\
0.421\end{array}$ & $\begin{array}{l}0.529 \\
0.512\end{array}$ \\
\hline
\end{tabular}

(Continued) 
Table 2 (Continued).

\begin{tabular}{|l|l|l|l|l|l|}
\hline Variables & Gray's Test & P-value & & \multicolumn{2}{|l|}{ Cumulative Incidence Function } \\
\cline { 3 - 6 } & & & I 2-mo & $\mathbf{3 6 - m o}$ & $\mathbf{6 0 - m o}$ \\
\hline Operation approach & & & & & \\
LLR & 0.594 & 0.441 & 0.222 & 0.575 & 0.575 \\
OLR & & & 0.182 & 0.421 & 0.514 \\
\hline
\end{tabular}

Note: Bold indicates statistically significant difference.

Abbreviations: $\mathrm{HBsAg}$, hepatitis B surface antigen; CA19-9, carbohydrate antigen 19-9; MVI, microvascular invasion; LLR, laparoscopic liver resection; OLR, open liver resection.

Table 3 Multivariable Analysis in Elderly Patients with iCCA

\begin{tabular}{|c|c|c|c|c|c|c|}
\hline \multirow[b]{2}{*}{ Variables } & \multicolumn{3}{|c|}{ Cox Regression Analysis } & \multicolumn{3}{|c|}{ Fine-Gray Regression Analysis } \\
\hline & HR & $95 \% \mathrm{Cl}$ & $P$ value & HR & $95 \% \mathrm{Cl}$ & $P$ value \\
\hline Age ( $\geq 70$ vs $<70$ years) & 1.706 & $1.101-2.645$ & 0.017 & 1.176 & $0.687-2.013$ & 0.560 \\
\hline Sex (female vs male) & 0.959 & $0.673-1.367$ & 0.817 & 0.781 & $0.520-1.173$ & 0.230 \\
\hline CAI9-9 (>29.5 vs $\leq 29.5 \mathrm{U} / \mathrm{mL})$ & 1.332 & $0.94 I-1.886$ & 0.106 & 1.283 & $0.875-1.862$ & 0.210 \\
\hline Maximum tumor size ( $>5$ vs $\leq 5 \mathrm{~cm}$ ) & 0.982 & $0.685-1.408$ & 0.982 & 1.082 & $0.711-1.643$ & 0.720 \\
\hline Tumor number (multiple vs single) & 1.314 & $0.902-1.914$ & 0.154 & 1.232 & $0.797-1.912$ & 0.340 \\
\hline Tumor encapsulation (incomplete vs complete) & 1.188 & $0.830-1.702$ & 0.346 & & & \\
\hline MVI (presence vs absence) & 1.699 & $|.093-2.64|$ & 0.019 & 1.843 & $1.138-2.962$ & 0.013 \\
\hline Macroscopic vascular invasion (presence vs absence) & 2.398 & $1.648-3.489$ & $<0.001$ & 2.405 & $1.602-3.591$ & $<0.001$ \\
\hline Satellite nodules (yes vs no) & & & & 1.463 & $0.90 \mathrm{I}-2.384$ & 0.120 \\
\hline Lymph node metastasis (yes vs no) & 4.059 & $2.733-6.028$ & $<0.001$ & 1.796 & $1.141-2.823$ & $0.0 \mathrm{II}$ \\
\hline
\end{tabular}

Note: Bold indicates statistically significant difference.

Abbreviations: $\mathrm{HBsAg}$, hepatitis B surface antigen; CA19-9, carbohydrate antigen 19-9; MVI, microvascular invasion; LLR, laparoscopic liver resection; OLR, open liver resection.

invasion. ${ }^{35}$ Given the increasing population of elderly people worldwide, geriatric patients constitute a large proportion of iCCA patients each year. Elderly patients often have comorbidities including metabolic and cardiovascular diseases, which can prevent them from receiving curative treatments because of the anesthetic and surgical risks involved. In studies of predictive factors for elderly patients, Cox regression on the event of interest would bring problems because a factor that increases the rate of the outcome event might not increase the risk of it in fact due to the high incidence of comorbidities in the elderly individuals. To date, there are few studies on iCCA in elderly patients undergoing surgery, which is a special group, and the prognostic factors of this group of patients after liver resection are rarely reported. Therefore, it is necessary to use the competing-risks model to address multiple end events in elderly iCCA patients. To the best of our knowledge, our study was the first one reported to date to apply the competing risk analysis model in the evaluation of prognostic factors in elderly patients with iCCA after liver resection based on a large number of elderly iCCA patients. In our study, we found that there was no significant difference in the extent of surgical resection between the youth group and the elderly group, indicating that patients' age had no influence on resectability or the extent of surgery. Liver resection for elderly iCCA patients is safe and feasible. In addition, our study found that elderly iCCA patients (age $\geq 60$ years) had comparable long-term outcomes with non-elderly iCCA patients (age $<60$ years) except that the $\geq 60$-years group had a relatively higher incidence of major complications. In addition to the cut-off of 60 years, we further differentiated the $\geq 60$-year group in $<70$ and $\geq 70$ years. In the Cox regression analysis, we found that (age $\geq 70$ years, HR, 1.706, 95\% CI, 1.101-2.645) was an independent risk factor affecting the prognosis of elderly iCCA patients, however, in fact, the Cox regression overestimated the impact of age risk on survival outcomes because competitive events were not taken into account. Our competing risk analysis indicated that age was not a statistically significant factor for iCCA specific survival. Different from the Cox regression analysis, we selected three independent 
Points

$\begin{array}{lllllllllll}0 & 10 & 20 & 30 & 40 & 50 & 60 & 70 & 80 & 90 & 100\end{array}$

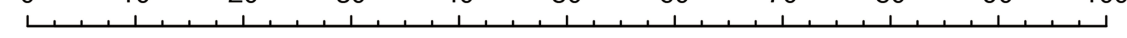

Microvascular invasion

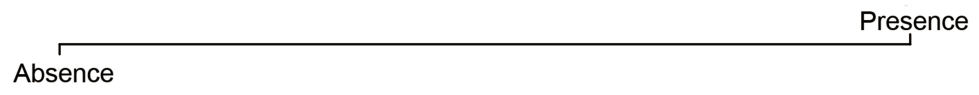

Macroscopic vascular invasion

Absence

Presence

\section{Lymph node metastasis}

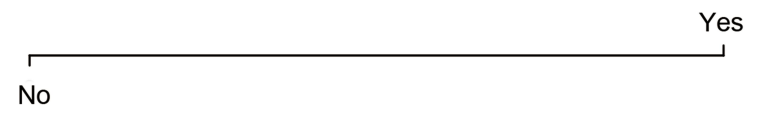

Total Points

\begin{tabular}{llllllllllllll}
\hline 0 & 20 & 40 & 60 & 80 & 100 & 120 & 140 & 160 & 180 & 200 & 220 & 240 & 260
\end{tabular}

Linear Predictor

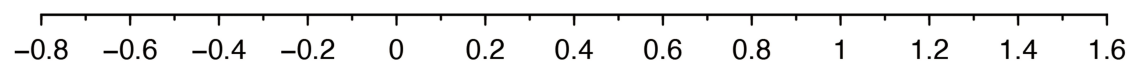

1-year survival Prob.

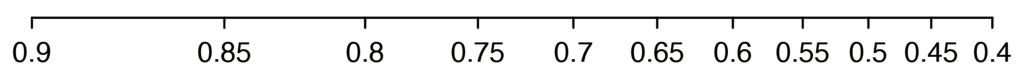

3-year survival Prob.

\begin{tabular}{lllllll}
\hline 0.7 & 0.6 & 0.5 & 0.4 & 0.3 & 0.2 & 0.1
\end{tabular}

5-year survival Prob.

\begin{tabular}{lllllllllllllllll}
\hline & 0.65 & 0.6 & 0.55 & 0.5 & 0.45 & 0.4 & 0.35 & 0.3 & 0.25 & 0.2 & 0.15 & 0.1 & 0.05
\end{tabular}

Figure 3 Competing risk nomogram predicting the I-year, 3-year and 5-year cumulative probabilities of death from cancer-specific mortality in elderly iCCA patients.

predictors (including MVI, MCI and LNM) through the competing risk analysis after controlling for other competing factors and included them in the prognostic nomogram. The established nomogram was derived from retrospectively collected data on 230 patients from singlecenter, showing favorable discrimination and calibration.
To assess the clinical usefulness of the nomogram. DCA was employed to determine whether nomogram-based decisions could improve patients' survival outcomes. We could find that our nomogram showed that the clinical net benefit gained from the competing risk model was higher than other iCCA clinical stages in the DCA. Furthermore, 


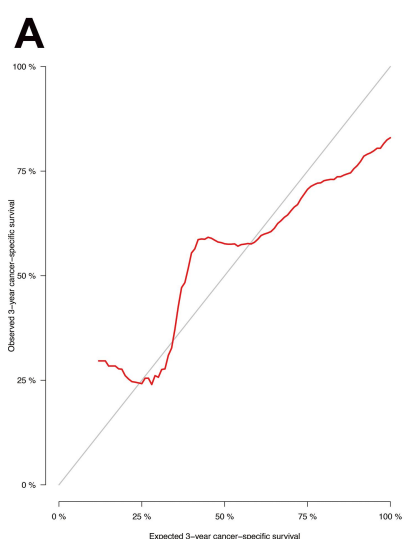

E

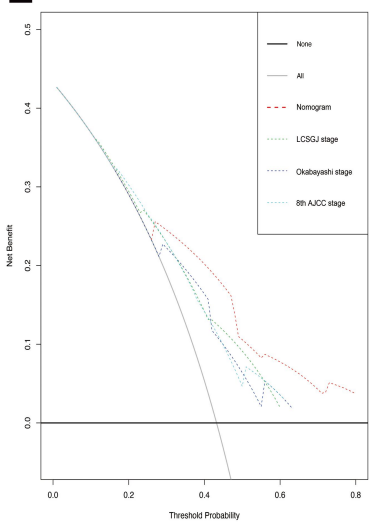

B

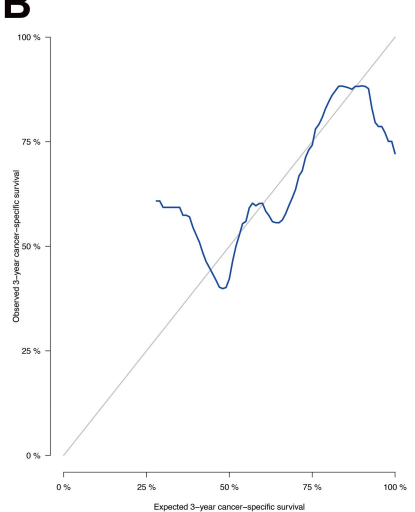

$\mathbf{F}$

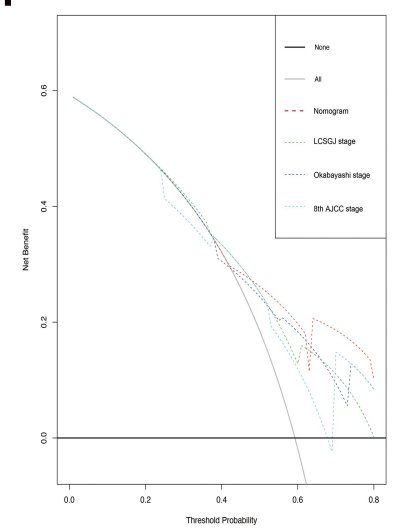

C

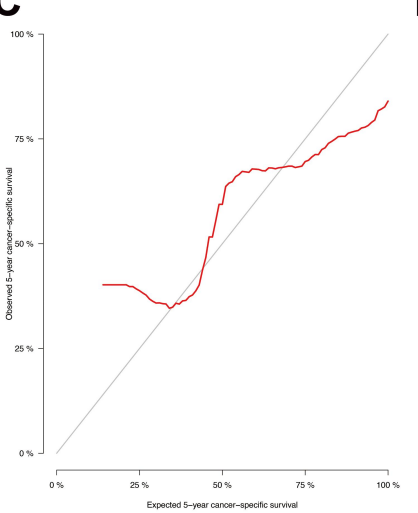

G

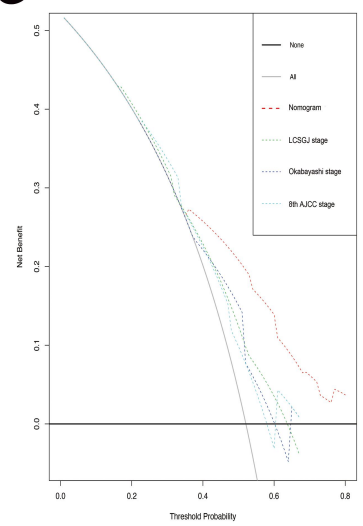

D

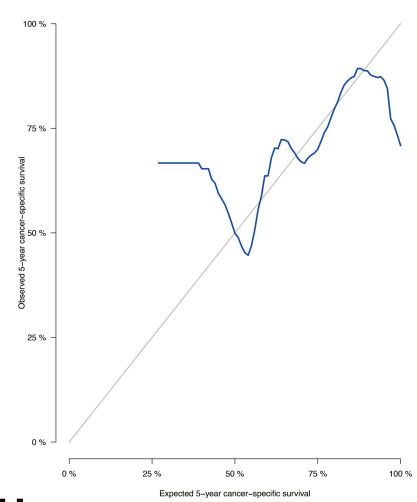

H

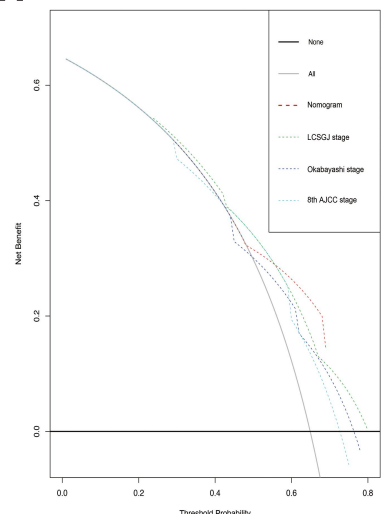

Figure 4 The 3-year and 5-year calibration curves for the training set ( $\mathbf{A}$ and $\mathbf{C}$, red) and validation set (B and $\mathbf{D}$, blue). The $\mathbf{X}$-axes represent the mean predicted mortality probability according to the prediction model. The Y-axes represent the observed cumulative incidence of mortality. The grey diagonal line indicates equality between the predicted and observed values. Decision curve analysis was used to compare the clinical net benefit of our nomogram with that of the Okabayashi staging system, the Liver Cancer Study Group of Japan (LCSGJ) staging system, and the 8th edition AJCC staging system in terms of the 3-year and 5-year survival of elderly iCCA patients in the training set $(\mathbf{E}$ and $\mathbf{G})$ and validation set $(\mathbf{F}$ and $\mathbf{H})$.

with the assistance of the nomogram, clinicians could successfully discriminate among different risk groups, thereby improving clinical decision making.

Many previous studies have shown MVI and LNM were strong indicators of worse outcomes among patients with iCCA after liver resection. ${ }^{36}$ In this study, it was found that the above factors were also important predictors in elderly iCCA patients. When MVI and LNM were present, the tumor was more aggressive and more likely to have intrahepatic metastasis and recurrence. Elevated CA19-9 levels are associated with poor prognosis due to higher tumor burden, ${ }^{37,38}$ However, in our multivariate analysis, CA19-9 was not an independent risk factor for survival, which may be related to the selection of cutoff values for CA19-9. Whether in Cox regression analysis or competitive risk analysis, tumor size was not a factor in evaluating prognostic outcomes in our study. This could partly explain why other iCCA classifications did not exhibit satisfactory discrimination in survival curves based on different stages. In recent years, studies have reported that radiofrequency ablation has potential application value for the treatment of $\mathrm{iCCA},{ }^{39}$ which may provide a new treatment option for elderly iCCA patients who could not tolerate major surgery or are unwilling to undergo liver resection. Whether adjuvant therapy could bring survival benefits to patients after iCCA surgery is still controversial. $^{40-42}$ In our study, it was found that adjuvant therapy had no effect on improving the prognosis of elderly iCCA patients, which may be related to the poor physical status of the elderly patients after surgery and the lower likelihood of these patients to tolerate adjuvant therapy. Moreover, age may influence a provider's choice to offer adjuvant therapy to elderly patients.

Although the main advantage of this present study is that it had a large enough sample size and established a nomogram to predict the prognosis of elderly iCCA patients based on the competitive risk model, there are still many limitations. First, the study came from a single- 
A

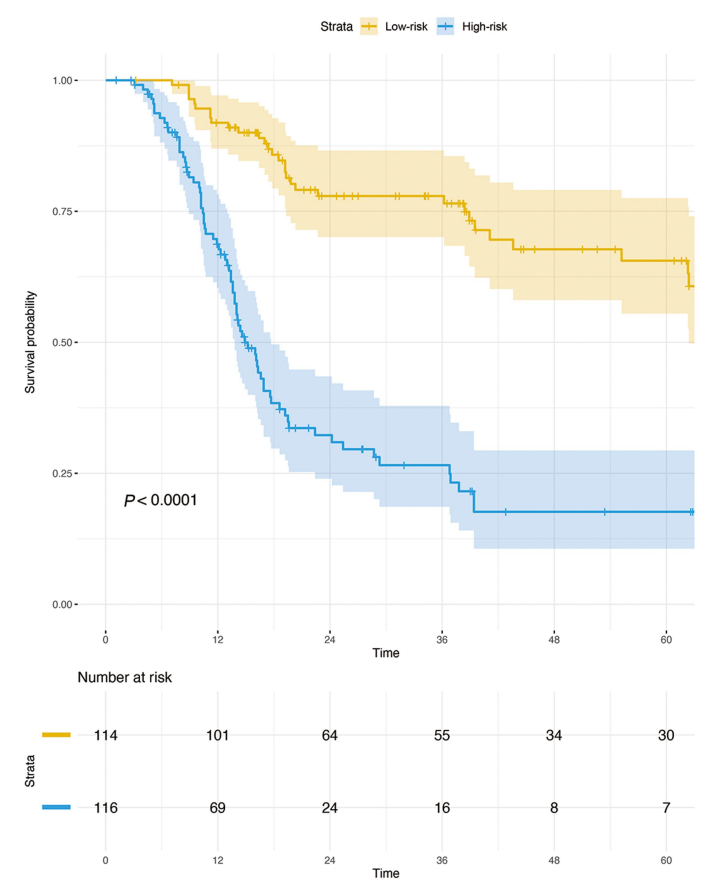

C

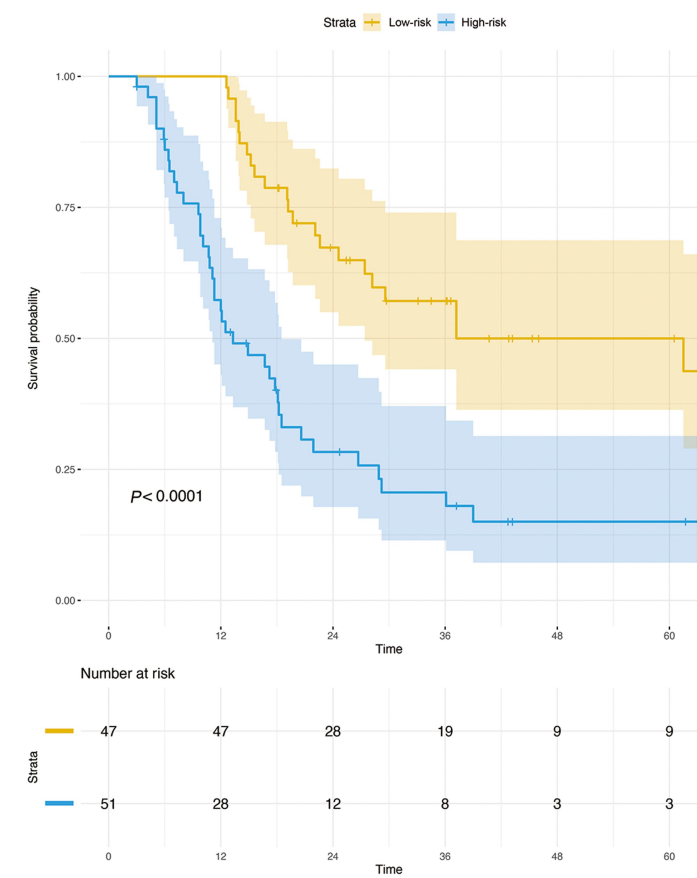

B

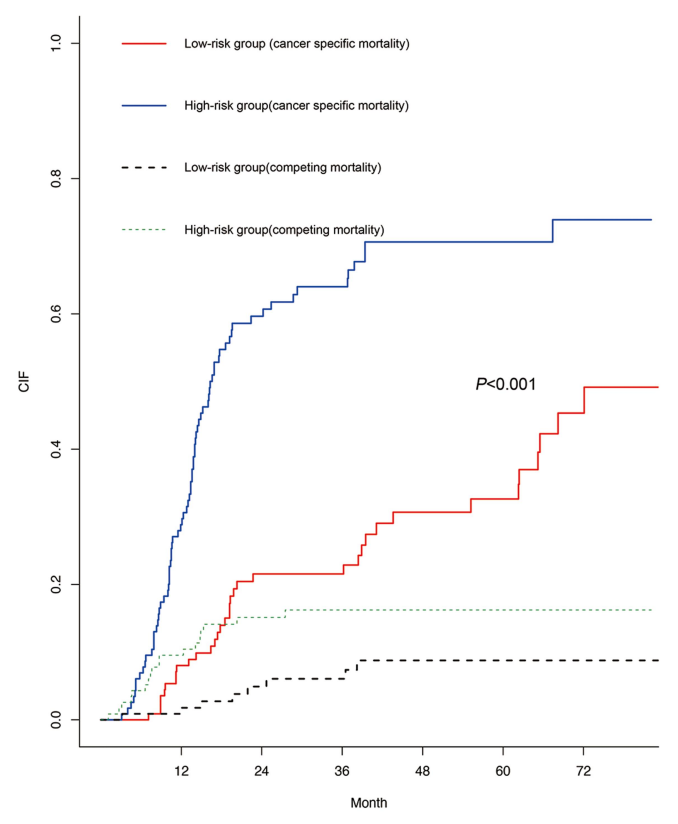

D

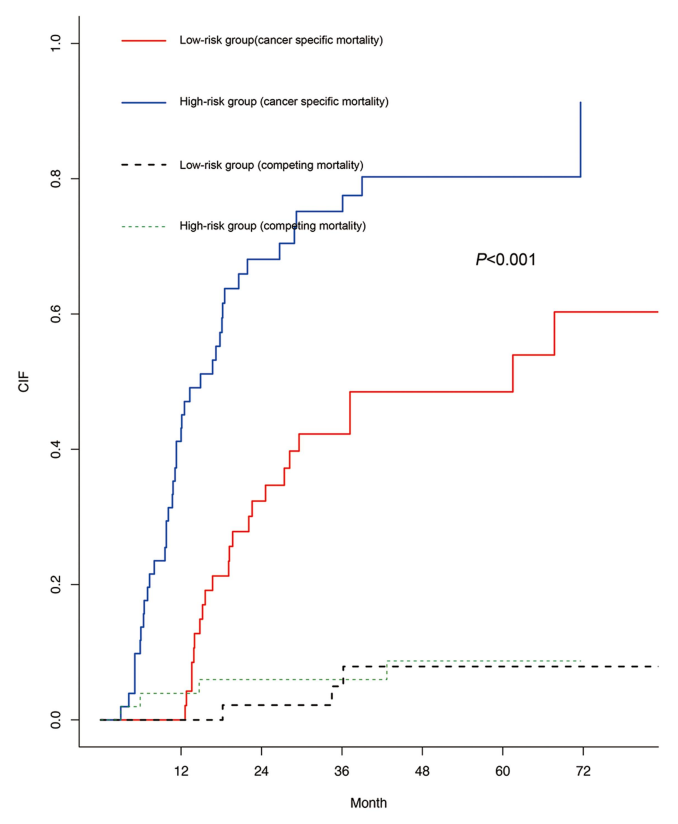

Figure $\mathbf{5}$ Kaplan-Meier analysis of overall survival (OS) between high-risk and low-risk groups for the training set and validation set (A and $\mathbf{C})$. Cumulative incidence function curves with the P-value of Gray's test between the high-risk and low-risk groups for the training set and validation set (B and $\mathbf{D})$.

institutional study with potential selection bias due to the characteristics of retrospective studies, Moreover, all patients were used to form the training set to develop the nomogram, and $70 \%$ of patients were randomly selected to serve as an internal validation set in this study. Although this is a generally accepted method for nomogram construction and validation, external validation based on other populations is still needed to estimate model accuracy. Second, our study object was iCCA patients undergoing liver resection, so we lack comparison with other treatment methods. 
In conclusion, we found that elderly iCCA patients undergoing liver surgery had comparable long-term CSS and OS rates that were comparable to those of non-elderly patients. As such, age was not a contraindication for liver resection in iCCA patients. When judging whether an iCCA patient can obtain survival benefits from surgery, we should consider the resectability of the tumor lesion and the patient's body status rather than simply age. Moreover, we constructed and validated a nomogram that could objectively and accurately predict the prognosis of elderly iCCA patients.

\section{Data Sharing Statement}

The data that support the findings of this study are available on request from the corresponding author upon reasonable request. The data are not publicly available due to privacy restrictions.

\section{Ethical Statement}

Every patient in our hospital after 2012 had signed the written informed consent form after admission. As for the patients before 2012, during the follow-up of outpatient clinic visits or phone call, we explained in detail the purpose of collecting patient clinical data and information with the patients and their legal guardians, verbal informed consent was obtained from all patients to use their clinical data for research purposes and noted in the record which was approved by the ethics committee of Sichuan University West China Hospital. The study was conducted in accordance with the Declaration of Helsinki. We keep patient medical information confidential.

\section{Acknowledgments}

We would like to acknowledge with gratitude the contribution of the colleagues of department of Liver Surgery, West China Hospital of Sichuan University.

\section{Author Contributions}

All authors made a significant contribution to the work reported, whether that is in the conception, study design, execution, acquisition of data, analysis and interpretation, or in all these areas; took part in drafting, revising or critically reviewing the article; gave final approval of the version to be published, have agreed on the journal to which the article has been submitted; and agree to be accountable for all aspects of the work.

\section{Funding}

This research was funded by the National Natural Science Foundation of China (No. 81770566), the Science and Technology Program of Sichuan Science and Technology Department (No. 2019YFS0029, 2019YFS0529), and Inner Mongolia Autonomous Region Applied Technology Research and Development Funding Plan (No. Kjt15sf05).

\section{Disclosure}

The authors declare that the research was conducted in the absence of any commercial or financial relationships that could be construed as a potential conflict of interest. All the authors disclose no conflicts.

\section{References}

1. Bertuccio P, Malvezzi M, Carioli G, et al. Global trends in mortality from intrahepatic and extrahepatic cholangiocarcinoma. J Hepatol. 2019;71(1):104-114. doi:10.1016/j.jhep.2019.03.013

2. Sirica AE, Gores GJ, Groopman JD, et al. Intrahepatic cholangiocarcinoma: continuing challenges and translational advances. Hepatology. 2019;69(4):1803-1815. doi:10.1002/hep.30289

3. Khan SA, Genus T, Morement H, et al. Global trends in mortality from intrahepatic and extrahepatic cholangiocarcinoma. J Hepatol. 2019;71 (6):1261-1262. doi:10.1016/j.jhep.2019.07.024

4. Pericleous M, Khan SA. Epidemiology of HPB malignancy in the elderly. Eur J Surg Oncol. 2020. doi:10.1016/j.ejso.2020.03.222

5. Bridgewater J, Galle PR, Khan SA, et al. Guidelines for the diagnosis and management of intrahepatic cholangiocarcinoma. $J$ Hepatol. 2014;60(6):1268-1289. doi:10.1016/j.jhep.2014.01.021

6. Choi SB, Kim KS, Choi JY, et al. The prognosis and survival outcome of intrahepatic cholangiocarcinoma following surgical resection: association of lymph node metastasis and lymph node dissection with survival. Ann Surg Oncol. 2009;16(11):3048-3056. doi:10.1245/ s10434-009-0631-1

7. Wright GP, Perkins S, Jones H, et al. Surgical resection does not improve survival in multifocal intrahepatic cholangiocarcinoma: a comparison of surgical resection with intra-arterial therapies. Ann Surg Oncol. 2018;25(1):83-90. doi:10.1245/s10434-0176110-1

8. Shaib YH, Davila JA, McGlynn K, et al. Rising incidence of intrahepatic cholangiocarcinoma in the United States: a true increase? J Hepatol. 2004;40(3):472-477. doi:10.1016/j.jhep.2003.11.030

9. Bartsch F, Baumgart J, Tripke V, et al. Resection of intrahepatic cholangiocarcinoma in elderly patients - is it reasonable? BMC Surg. 2019;19(1):157. doi:10.1186/s12893-019-0620-7

10. Vitale A, Spolverato G, Bagante F, et al. A multi-institutional analysis of elderly patients undergoing a liver resection for intrahepatic cholangiocarcinoma. J Surg Oncol. 2016;113(4):420-426. doi: $10.1002 /$ jso. 24148

11. Gooley TA, Leisenring W, Crowley J, et al. Estimation of failure probabilities in the presence of competing risks: new representations of old estimators. Stat Med. 1999;18(6):695-706. doi:10.1002/(SICI) 1097-0258(19990330)18:6<695::AID-SIM60>3.0.CO;2-O

12. Putter H, Fiocco M, Geskus RB. Tutorial in biostatistics: competing risks and multi-state models. Stat Med. 2007;26(11):2389-2430. doi:10.1002/sim. 2712

13. Carmona R, Zakeri K, Green G, et al. Improved method to stratify elderly patients with cancer at risk for competing events. $J$ Clin Oncol. 2016;34(11):1270-1277. doi:10.1200/JCO.2015.65.0739 
14. Janssen-Heijnen ML, Houterman S, Lemmens VE, et al. Prognostic impact of increasing age and co-morbidity in cancer patients: a population-based approach. Crit Rev Oncol Hematol. 2005;55 (3):231-240. doi:10.1016/j.critrevonc.2005.04.008

15. Mathers CD, Stevens GA, Boerma T, et al. Causes of international increases in older age life expectancy. Lancet. 2015;385 (9967):540-548. doi:10.1016/S0140-6736(14)60569-9

16. Austin PC. An introduction to propensity score methods for reducing the effects of confounding in observational studies. Multivariate Behav Res. 2011;46(3):399-424. doi:10.1080/00273171.2011.568786

17. Amin MB, Greene FL, Edge SB, et al. The eighth edition AJCC cancer staging Manual: continuing to build a bridge from a population-based to a more "personalized" approach to cancer staging. CA Cancer J Clin. 2017;67(2):93-99. doi:10.3322/ caac. 21388

18. Okabayashi T, Yamamoto J, Kosuge T, et al. A new staging system for mass-forming intrahepatic cholangiocarcinoma: analysis of preoperative and postoperative variables. Cancer. 2001;92 (9):2374-2383. doi:10.1002/1097-0142(20011101)92:9<2374::AIDCNCR1585>3.0.CO;2-L

19. Yamasaki S. Intrahepatic cholangiocarcinoma: macroscopic type and stage classification. J Hepatobiliary Pancreat Surg. 2003;10 (4):288-291. doi:10.1007/s00534-002-0732-8

20. Dahiya D, Wu TJ, Lee CF, et al. Minor versus major hepatic resection for small hepatocellular carcinoma (HCC) in cirrhotic patients: a 20-year experience. Surgery. 2010;147(5):676-685. doi:10.1016/j. surg.2009.10.043

21. Dindo D, Demartines N, Clavien PA. Classification of surgical complications: a new proposal with evaluation in a cohort of 6336 patients and results of a survey. Ann Surg. 2004;240(2):205-213. doi:10.1097/01.sla.0000133083.54934.ae

22. Onodera T, Goseki N, Kosaki G. [Prognostic nutritional index in gastrointestinal surgery of malnourished cancer patients]. Nihon Geka Gakkai Zasshi. 1984;85(9):1001-1005.

23. Wang J, Bo X, Li M, et al. Prediction efficacy for clinical outcome of prognostic nutritional index in patients with resectable biliary tract cancer depends on sex and obstructive jaundice status. Ann Surg Oncol. 2020. doi:10.1245/s10434-020-08728-8

24. Pinato DJ, North BV, Sharma R. A novel, externally validated inflammation-based prognostic algorithm in hepatocellular carcinoma: the prognostic nutritional index (PNI). $\mathrm{Br} J$ Cancer. 2012;106(8):1439-1445. doi:10.1038/bjc.2012.92

25. Hsu CY, Lee YH, Hsia CY, et al. Performance status in patients with hepatocellular carcinoma: determinants, prognostic impact, and ability to improve the barcelona clinic liver cancer system. Hepatology. 2013;57(1):112-119. doi:10.1002/hep.25950

26. Zhang X, Zhang MJ, Fine J. A proportional hazards regression model for the subdistribution with right-censored and left-truncated competing risks data. Stat Med. 2011;30(16):1933-1951. doi:10.1002/ $\operatorname{sim} .4264$

27. Haller B, Schmidt G, Ulm K. Applying competing risks regression models: an overview. Lifetime Data Anal. 2013;19(1):33-58. doi:10.1007/s10985-012-9230-8

28. Harrell FE, Lee KL, Mark DB. Multivariable prognostic models: issues in developing models, evaluating assumptions and adequacy, and measuring and reducing errors. Stat Med. 1996;15(4):361-387. doi:10.1002/ (SICI)1097-0258(19960229)15:4<361::AID-SIM168>3.0.CO;2-4
29. Pencina MJ, D'Agostino RB. Overall C as a measure of discrimination in survival analysis: model specific population value and confidence interval estimation. Stat Med. 2004;23(13):2109-2123. doi: $10.1002 /$ sim. 1802

30. Vickers AJ, Cronin AM, Elkin EB, et al. Extensions to decision curve analysis, a novel method for evaluating diagnostic tests, prediction models and molecular markers. BMC Med Inform Decis Mak. 2008;8:53. doi:10.1186/1472-6947-8-53

31. Vickers AJ, Elkin EB. Decision curve analysis: a novel method for evaluating prediction models. Med Decis Making. 2006;26 (6):565-574. doi:10.1177/0272989X06295361

32. Southern DA, Faris PD, Brant R, et al. Kaplan-Meier methods yielded misleading results in competing risk scenarios. J Clin Epidemiol. 2006;59(10):1110-1114. doi:10.1016/j. jclinepi.2006.07.002

33. Jepsen P, Vilstrup H, Andersen PK. The clinical course of cirrhosis: the importance of multistate models and competing risks analysis. Hepatology. 2015;62(1):292-302. doi:10.1002/hep.27598

34. Andersen PK, Geskus RB, de Witte T, et al. Competing risks in epidemiology: possibilities and pitfalls. Int J Epidemiol. 2012;41 (3):861-870. doi:10.1093/ije/dyr213

35. Shimada M, Yamashita Y, Aishima S, et al. Value of lymph node dissection during resection of intrahepatic cholangiocarcinoma. $\mathrm{Br}$ $J \quad$ Surg. 2001;88(11):1463-1466. doi:10.1046/j.00071323.2001.01879.x

36. Wang T, Kong J, Yang X, et al. Clinical features of sarcomatoid change in patients with intrahepatic cholangiocarcinoma and prognosis after surgical liver resection: a propensity score matching analysis. J Surg Oncol. 2020;121(3):524-537. doi:10.1002/jso.25815

37. Luo X, Yuan L, Wang Y, et al. Survival outcomes and prognostic factors of surgical therapy for all potentially resectable intrahepatic cholangiocarcinoma: a large single-center cohort study. $J$ Gastrointest Surg. 2014;18(3):562-572. doi:10.1007/s11605-013-2447-3

38. Yamada T, Nakanishi Y, Okamura K, et al. Impact of serum carbohydrate antigen 19-9 level on prognosis and prediction of lymph node metastasis in patients with intrahepatic cholangiocarcinoma. J Gastroenterol Hepatol. 2018;33(9):1626-1633. doi:10.1111/ jgh. 14124

39. Brandi G, Rizzo A, Dall'Olio FG, et al. Percutaneous radiofrequency ablation in intrahepatic cholangiocarcinoma: a retrospective single-center experience. Int $J$ Hyperthermia. 2020;37(1):479-485. doi: $10.1080 / 02656736.2020 .1763484$

40. Cloyd JM, Ejaz A, Pawlik TM. The landmark series: intrahepatic cholangiocarcinoma. Ann Surg Oncol. 2020;27(8):2859-2865. doi:10.1245/s10434-020-08621-4

41. Lee J, Park SH, Chang HM, et al. Gemcitabine and oxaliplatin with or without erlotinib in advanced biliary-tract cancer: a multicentre, open-label, randomised, phase 3 study. Lancet Oncol. 2012;13 (2):181-188. doi:10.1016/S1470-2045(11)70301-1

42. Neoptolemos JP, Palmer DH, Ghaneh P, et al. Comparison of adjuvant gemcitabine and capecitabine with gemcitabine monotherapy in patients with resected pancreatic cancer (ESPAC-4): a multicentre, open-label, randomised, phase 3 trial. Lancet. 2017;389 (10073):1011-1024. doi:10.1016/S0140-6736(16)32409-6 


\section{Publish your work in this journal}

Cancer Management and Research is an international, peer-reviewed open access journal focusing on cancer research and the optimal use of preventative and integrated treatment interventions to achieve improved outcomes, enhanced survival and quality of life for the cancer patient.

The manuscript management system is completely online and includes a very quick and fair peer-review system, which is all easy to use. Visit http://www.dovepress.com/testimonials.php to read real quotes from published authors. 